\title{
Encore et à nouveau sur la neurogliobiologie
}

Le rôle des cellules macrogliales - et plus précisément des astrocytes dans le fonctionnement des circuits neuronaux devient de plus en plus évident au fur et à mesure que se multiplient les études... qui le recherchent ! Parmi les travaux récents, deux sont particulièrement importants en ce qu'ils démontrent une intervention directe de l'activité astrocytaire sur la concentration cytosolique de calcium de neurones voisins, c'est-à-dire sur l'un des éléments clés de leur système de signalisation.

Maiken Nedergaard (Cornell University, NY, USA) a travaillé sur des cocultures neurones-astrocytes en utilisant la propriété de ces derniers déjà décrite dans médecine/sciences $\left(\mathrm{m} / \mathrm{s} n^{\circ} 6\right.$, vol. 6, p. 597) - de développer une vague d'augmentation de calcium intracellulaire se propageant en nappe à partir d'un site de stimulation focal [1]. Dans des cultures d'astrocytes ou de neurones purifiés, une stimulation électrique focale active une augmentation du calcium intracellulaire dans les cellules situées au site de stimulation. Seuls les astrocytes voient secondairement se propager la vague calcique. Or lorsque l'auteur a réalisé des cocultures, il a observé que les neurones inclus dans la zone soumise à la propagation de la vague calcique astrocytaire subissent eux aussi une élévation importante de la concentration de calcium intracellulaire. Sachant que dic telles vagues calciques existent in vivo, il est licite de considérer que l'état d'activation des réseaux astrocytaires d'une région cérébrale est un déterminant important de l'excitabilité neuronale dans la même zone. Les constantes de temps de ces vagues calciques sont de l'ordre de la minute, voire de la dizaine de minutes. Des modifications du fonctionnement astrocytaire pourraient donc, de fait, être à l'origine de phénomènes comportementaux de durées moyennes.

Les travaux de Philip Haydon et de son équipe (Iowa State University, IO, USA) vont dans le même sens d'une intervention directe des astrocytes sur la signalisation interneuronale en démontrant l'existence d'une influence glioneuronale glutamatergique [2]. Les auteurs ont utilisé le fait que la bradykinine accroît la concentration cytosolique de calcium et la libération de glutamate d'astrocytes en culture (et non de neurones) pour examiner en coculture l'effet de cette libération astrocytaire sur des neurones. Comme espéré, cette libération a provoqué une activation neuronale que les auteurs ont pu bloquer en ajoutant un antagoniste glutamatergique et même, plus spécifiquement, un antagoniste NMDA. Les auteurs ont raffiné ensuite cette démonstration en utilisant la photostimulation pour activer de façon indépendante l'un des astrocytes dans la coculture. Cette monostimulation déclenchant une vague calcique dont ils ont pu suivre la progression, les auteurs ont ainsi vérifié l'existence d'une augmentation de calcium cytosolique dans les neurones voisins et le blocage de cet effet par un antagoniste glutamatergique.

On sait depuis quelques années que les astrocytes peuvent être stimulés par le glutamate libéré par des prolongements neuronaux $\left(\mathrm{m} / \mathrm{s} n^{\circ} 7\right.$, vol. 5, p. 511) et il a été montré récemment que cette stimulation peut déclencher des vagues calciques [3], comme le peuvent de nombreux neurotransmetteurs agissant sur leurs récepteurs spécifiques portés par les astrocytes $\left(\mathrm{m} / \mathrm{s} n^{\circ} 8\right.$, vol. 7, p. 766). Les vagues de calcium assurant le transfert d'une information ponctuelle dans un espace incomparablement plus large que celui défini par un contact synaptique, on peut envisager que les circuits astrocytaires peuvent ainsi participer dans le cerveau à des modulations régionales de l'activité neuronale.

Les travaux consacrés aux interactions physiologiques neuronogliales en sont encore, sans doute, à leurs balbutiements. Le tableau qui se dessine de façon toujours assez floue est déjà, toutefois, extraordinairement captivant, car il superpose aux systèmes de transmission interneuronaux ultra-rapides et hyperlocalisés des systèmes d'" ambiance ", faisant intervenir des réseaux astrocytaires, dont les caractéristiques temporelles (semi-lentes) et spatiales (régionales) sont très différentes. La mise au jour de la neuroplasticité nous disait qu'un réseau neuronal n'était pas immuable; la neurogliobiologie nous affirme, en plus, qu'il ne fonctionne jamais tout seul.

M.P.

1. Nedergaard M. Direct signaling from astrocytes to neurons in cultures of mammalian brain cells. Srience $1994 ; 263: 1768-71$

2. Parpura V', Basarsky TA, I.iu F, Jeftimja K, Jeftimja S, Haydon PG. Glutamate-mediated astrocyte-neuron signalling. Nature $1994 ; 369$ : 744-7.

3. Dani JW', Chernjavisky A, Smith SJ. Neuronal activity triggers calcium waves in hippocampal astrocyte networks. Neuron $1992 ; 8: 429-40$. 